\title{
The Effect of Milling Speed on Particle Size and Morphology of Cu25W Composite Powder
}

\author{
S. BIYIK ${ }^{a, *}$, M. AYDIN ${ }^{b}$ \\ ${ }^{a}$ Karadeniz Technical University, Department of Metallurgical and Materials Engineering, 61080 Trabzon, Turkey \\ ${ }^{b}$ Karadeniz Technical University, Department of Mechanical Engineering, 61080 Trabzon, Turkey \\ In this study, the effect of milling speed on particle size and morphology of Cu25W composite powder produced \\ by high-energy ball milling was investigated. For this aim, commercial elemental copper and tungsten powders \\ were milled in a planetary-type ball mill for different milling durations. Ball-to-powder weight ratio was selected \\ as 10:1. Three different milling speeds, namely 200, 300, and $400 \mathrm{rpm}$ were used throughout the tests. In order to \\ avoid agglomeration and to decrease the tendency of cold welding among powder particles, stearic acid in amount \\ of 2 wt.\% was used as a process control agent. The morphological and microstructural evolution of the milled \\ powders was evaluated by scanning electron microscopy. In addition, the variation of particle size and powder \\ morphology as a function of milling duration was determined. As a result of this effort, the milling duration was \\ found to have strong effect on the structural evolution of the powder, and the optimum particle size as a function \\ of milling speed was determined.
}

DOI: 10.12693 /APhysPolA.127.1255

PACS: $81.20 . \mathrm{Ev}$

\section{Introduction}

Mechanical alloying (MA) is a powder processing technique which involves loading the blended elemental or prealloyed powder particles along with the grinding medium in a vial and subjecting them to heavy deformation [1]. It allows to synthesize a wide variety of equilibrium and non-equilibrium alloy phases, and composites. It is also advantageous to obtain fine and homogeneous dispersion of brittle reinforcement phase in the ductile matrix. MA technology has been utilized in a variety of applications such as composites, superconductors, biomaterials, tribological materials, light metals, nickel-base superalloys, metastable phases and magnetic materials [2].

MA process includes repeated cold welding, fracturing and rewelding of powder particles in a high-energy ball mill. Cold welding and fracturing are the main events occurring during the milling operation, and it is essential to establish a balance between cold welding and fracturing for a successful milling. Depending upon the nature of the powder being milled (ductile or brittle), utilization of some surface additives called process control agent (PCA) is necessary to make this happen, especially when the powder mix involves a substantial fraction of a ductile component [3]. Therefore, the influence of the type and amount of PCA on the powder morphology should be carefully studied at first. Apart from this, MA involves optimization of a number of variables to achieve the desired product phase and/or microstructure. The most important parameters affecting the final constitution of the powder are the type of mill, milling container,

* corresponding author; e-mail: serkanbiyik@ktu.edu.tr milling speed, milling time, type, size, and size distribution of the grinding medium, extent of filling the vial, ball-to-powder weight ratio (BPR), milling atmosphere, $\mathrm{PCA}$, and temperature of milling [4].

The milling speed is one of the most important variables to be considered in MA experiments. It should be carefully optimized to obtain maximal collision energy. The energy input exerted on the powder by ball milling depends upon how fast the mill rotates. However, the maximum speed employed is somewhat limited by the design of the mill. It should also be emphasized that the maximal speed of rotation is of big importance on particle size reduction and morphology of the powders being milled. In this regard, the milling speeds lower or higher than the optimal value have some drawbacks. For example, very low rotational speeds lead to very long periods of milling and a large inhomogeneity in the alloy because of inadequate kinetic energy input [2]. Similarly, rotational speeds higher than the optimal value cause the grinding balls to be pinned to the inner walls of the vial and not to fall down to exert any impact force. Therefore, fracturing efficiency will be lower in such case. Apart from this, temperature rise generated by high speeds is another limitation for ball milling process. This may be beneficial in some cases where diffusion is required to promote homogenization and/or alloying in the powders. However, in some cases, the increase in temperature may lead to acceleration of transformation processes and formation of some undesirable phases, and powder contamination. In addition, increased milling speed may cause excessive wear of the milling tools, both grinding medium and container, which propagates powder contamination. Besides, increasing milling speed leads to higher degree of plastic deformation which is the source of increase in cold welding, and some of the powder get stuck to the inner walls of the vial. This results in a decrease in the 
powder yield [1]. To overcome the above-mentioned problems, the maximal speed should be just below the critical value. Thereby, the maximal collision energy will be provided in respect to maximal height where the balls fall down.

The methodology used in this study is based upon the optimization of the production of electrical contact materials. Contact material has a major influence on the performance of relays, contactors, and other switching devices. Silver $(\mathrm{Ag})$ based composite contact materials are frequently used in the electrical industry [5-10]. These include powder metal combinations which ordinarily cannot be achieved by alloying [11]. On the other hand, commercially pure copper $(\mathrm{Cu})$ has some benefits such as high electrical and thermal conductivity, ease of fabrication, low cost and plentiful supply as compared to precious metals, and these features make it an obvious choice as a contact material [12]. However, $\mathrm{Cu}$ has also some drawbacks such as poor resistance to oxidation and corrosion [13]. To improve the arc-erosion performance of electrical contact materials, refractory metals, such as tungsten (W) and molybdenum having high melting and boiling points, and excellent resistance to arc-erosion are frequently used in combination with $\mathrm{Ag}$ or $\mathrm{Cu}$ in applications involving severe arcing and welding. $\mathrm{Cu}$ can be used in contact applications either as the pure metal (applications where arcing and welding are not severe), or in the form of alloys or compounds made by powder metallurgy. Therefore, different types of $\mathrm{Cu}^{-}$ based composite electrical contact materials have been developed [14, 15]. Cu-based electrical contact materials are used in a variety of applications, such as arcing contacts in oil switches, current carrying contacts, vacuum interrupter, oil-circuit breakers, arcing tips, contactors, vacuum switches, automotive starters, instruments, fuel pumps, welding machines, industrial truck motors, automotive heaters, antenna motors and generators [11]. Much work has previously been done in production and evaluation of $\mathrm{Cu}$-based contact materials in terms of electrical performance [16-19]. The electrical performance of the contacts is directly related to the material parameters such as composition of the constituent powder mixtures, homogeneity, powder processing technique, compaction and sintering routes, and final surface treatments as well as the electrical parameters. The fine and homogeneous dispersion of refractory metals or metal oxides within the matrix enables to achieve contacts having superior performance as compared to that of conventional ones. Apart from the switching applications, there have also been other studies and attempts to synthesize $\mathrm{Cu}$ and/or W composites by MA technique [20-24].

Due to the reasons mentioned above, the milling speed as a function of milling duration should be carefully optimized for a given set of experimental conditions. For this aim, the effect of milling speed on particle size and morphology of $\mathrm{Cu} 25 \mathrm{~W}$ composite powder was investigated. This work may also be called as a pre-study for manufacturing of electrical contacts.

\section{Materials and method}

In this study, $\mathrm{Cu}$ was used as the matrix material and $\mathrm{W}$ as the reinforcement. As starting materials, elemental $\mathrm{Cu}$ having particle size of $44 \mu \mathrm{m}$ and purity of $99 \%$ and $\mathrm{W}$ having average particle size (APS) of $12 \mu \mathrm{m}$ and purity of $99.9 \%$ powders were used for ball milling experiments. Both $\mathrm{Cu}$ and $\mathrm{W}$ powders were supplied by Alfa Aesar Corporation. The morphology of the starting powders was investigated by means of scanning electron microscopy (SEM) on a Zeiss Evo LS 10 model microscope (Fig. 1). Particle size distribution of the starting $\mathrm{Cu}$ powder was analyzed using Malvern Instruments Laser Diffractometer Mastersizer 2000 (Fig. 2). Planetary-type ball mill, Fritsch Pulverisette 6, was used to carry out milling experiments. Here, it should be emphasized that it may not be possible to change the milling speed in all types of mills. Planetary-type ball mill used in this study is capable of altering the speed of rotation of the rotating disk and the containers. In addition, reversing operation enables to change the direction of rotation of the planetary ball mill. This allows variation of the impact energy acquired by the balls. Both milling container (vial) and grinding balls are made of tungsten carbide (WC). The diameter of each ball is $10 \mathrm{~mm}$.

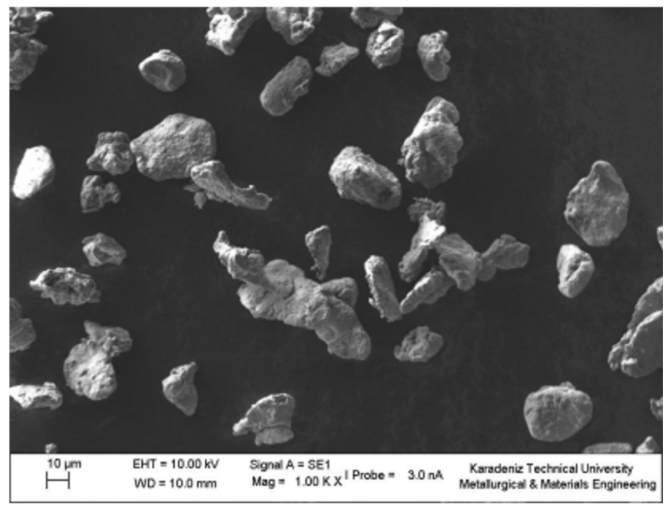

(a)

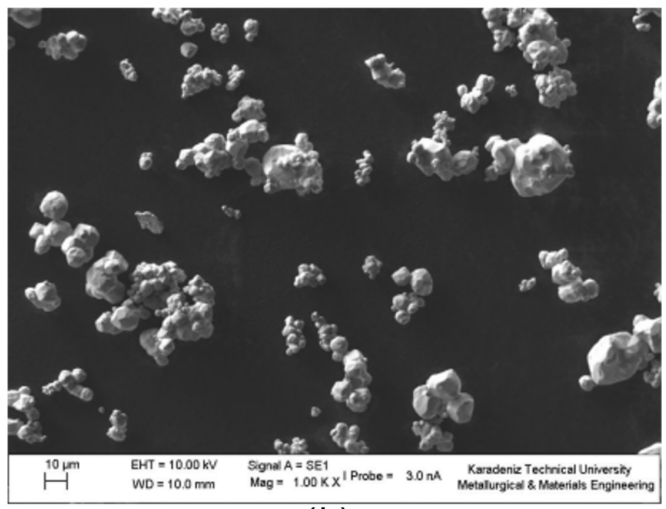

(b)

Fig. 1. Morphology of as-received powders (a) $\mathrm{Cu}$ powder, (b) $\mathrm{W}$ powder.

W powders were added to $\mathrm{Cu}$ powders in the amount of 25 wt.\%. The chemical composition of the composite 


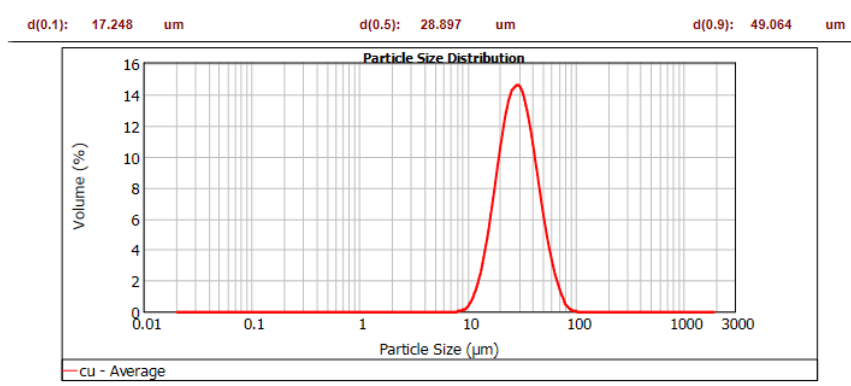

Fig. 2. Particle size distribution curve of starting $\mathrm{Cu}$ powder.

powder, namely the percentages of both matrix and reinforcement was kept constant in each experiment. Stearic acid in the amount of 2 wt.\% was used as a PCA. The milling was carried out at various milling speeds, namely 200, 300, and $400 \mathrm{rpm}$, and BPR was selected as 10:1. The powders were ball milled under the aforementioned test conditions by altering the milling speed for each test. The whole experiment may be subdivided into three separate experiments due to the differences of the milling speed. In this regard, the starting powders were ballmilled separately for up to $25 \mathrm{~h}$. For each test, having different milling speed, the fresh powder charge was installed into the vial. In addition, to prevent overheating of the grinding medium, the milling process was interrupted for at least 30 minutes after certain milling durations, until the room temperature ensured. All milling conditions for the whole experiment are given in Table I. After each test duration, which are listed in the table, the powder samples were withdrawn for particle size measurements. The variation of APS $\left(d_{50}\right)$ with milling duration was also investigated by SEM.

Milling parameters used to investigate the effect of milling speed on particle size and TABLE I morphology

\begin{tabular}{l|l}
\hline \hline Type of Mill & Planetary Ball Mill \\
Grinding Medium & WC balls $(\Phi=10 \mathrm{~mm})$ \\
Amount of PCA [wt.\%] & 2 \\
Milling Energy/Speed [rpm] & 200,300, and 400 \\
Milling Time [h] & $0.5,2,4,7,10,16,25$ \\
BPR & $10: 1$ \\
Milling Atmosphere & Air \\
Chamber Temperature $\left[{ }^{\circ} \mathrm{C}\right]$ & Room Temperature \\
Chamber Capacity $[\mathrm{ml}]$ & 225 \\
\hline
\end{tabular}

The optimization of the production of $\mathrm{Cu} 25 \mathrm{~W}$ composite powder was achieved by comparing these separate experiments with respect to particle size reduction and corresponding morphology.

\section{Results and discussion}

The initial morphologies of both $\mathrm{Cu}$ and $\mathrm{W}$ powders are shown in Fig. 1a and 1b, respectively. It can be seen from the figure that $\mathrm{Cu}$ powders have irregular shape whereas $\mathrm{W}$ powders have angular shape from a morphological point of view. To simplify and better understand the observations, the whole experiment was sub-divided into three process codes as seen in Table II. The shapes of the particles and corresponding morphologies, for the increasing milling duration, are presented in Table III and in Fig. 3 and 4, respectively. Figure 3 shows the SEM images of the powders milled at various milling speeds for different milling durations, namely $0.5,2,4$ and $7 \mathrm{~h}$. It is clear from the figure that powder particles had undergone morphological changes with the increasing milling duration. In the initial stages of milling, namely $0.5 \mathrm{~h}$, the ductile $\mathrm{Cu}$ powders got flattened by the ball-powderball collisions and flake morphology was partly observed in the powders milled at 300 and $400 \mathrm{rpm}$, Fig. 3b and 3c. However, $0.5 \mathrm{~h}$ of milling duration was not sufficient to obtain flake morphology in the powders milled at $200 \mathrm{rpm}$ as can be seen from Fig. 3a. It is clear from the same Figure that reducing milling speed caused the decrease of plastic deformation, and the powders have exhibited less change in morphology. Therefore, the powder particles remained irregular. As the process continues, severe deformation between the powder particles was observed. Thereby, distinctive flake morphology was seen in P2 and P3 processes after milled for $2 \mathrm{~h}$ (Fig. 3e and $3 \mathrm{f}$ ). On the other hand, flake morphology was partially achieved for $\mathrm{P} 1$ process at the same milling duration (Fig. 3d). In time, the particles get harder and their ability to withstand deformation without fracturing decreases. Namely, milling duration of $4 \mathrm{~h}$ is critical point in terms of fragmentation, especially for P2 process (Fig. $3 \mathrm{~h}$ ).

TABLE II

The milling speed with respect to the codes of process.

\begin{tabular}{l|l}
\hline \hline P1 & 200 \\
P2 & 300 \\
P3 & 400 \\
\hline
\end{tabular}

The variation of particle size may be clearly visible even on a macroscopic scale for some time intervals. Here, it should be emphasized that $4 \mathrm{~h}$ of milling duration is sufficient for $\mathrm{P} 2$ process, as fracturing begins in this period, while the same phenomenon occurs at later stages of MA for P3 process. Therefore, this critical point moved up to $7 \mathrm{~h}$ for P3 process (Fig. 3l). On the other hand, it could be seen that reducing milling speed decreased the ball-to-powder collisions and reduced the efficiency. Namely, rate of fracturing is fairly low for $\mathrm{P} 1$ process as seen in Fig. 3j.

Figure 4 shows the SEM images of the powders milled for 10,16 and $25 \mathrm{~h}$ with respect to three different milling speeds. It can be seen from Fig. 4a-4c that deformation and flattening of the soft $\mathrm{Cu}$ matrix are dominate the fracturing. Hence, the flake powders are the majority fraction in $\mathrm{P} 1$ process, even after milling for $25 \mathrm{~h}$ (Fig. 4c). On the other hand, distinctive semi-equiaxial morphology was observed in $\mathrm{P} 2$ and $\mathrm{P} 3$ processes at the later stages of milling experiments, namely 10 and $16 \mathrm{~h}$, respectively (Figs. $4 \mathrm{~d}$ and $4 \mathrm{~h}$ ). Comparing P2 and 
P3 processes, grain refinement of both $\mathrm{Cu}$ matrix and $\mathrm{W}$ reinforcement was achieved and more homogeneous distribution of the brittle $\mathrm{W}$ phase was observed in $\mathrm{P} 2$ process. However, broad size distribution of powders was observed in P3 process (Fig. 4h), as impact energy of the balls decreased beyond the maximal milling speed value. As can be seen from Fig. 4g-4i, fracturing efficiency de- creased beyond the maximal speed value. For speeds greater than the optimum, W particles exposed to less deformation and consequently they remain coarser and flake like in the microstructure (Fig. 4h). Similarly, the milling speeds lower than the optimum caused to exert lower impact forces on the powders, and $\mathrm{W}$ particles remained coarse as well (Fig. 4b).

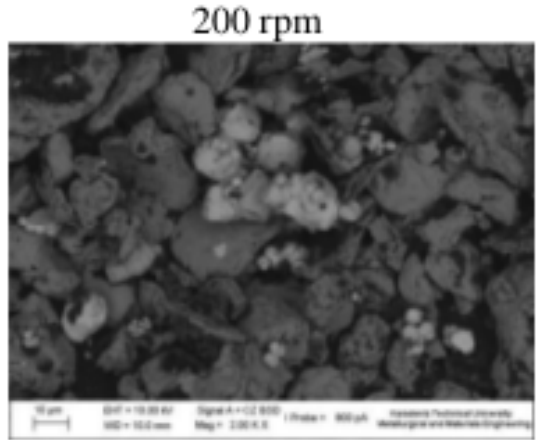

(a)

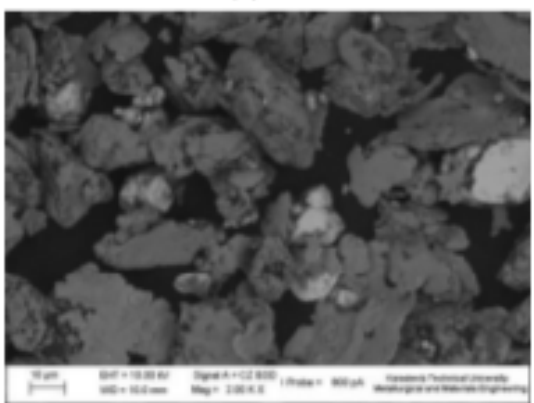

(d)

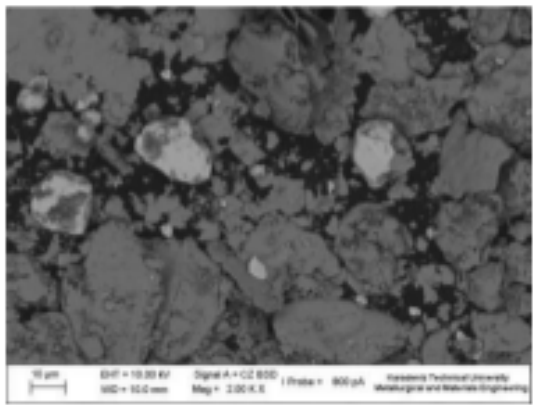

(g)

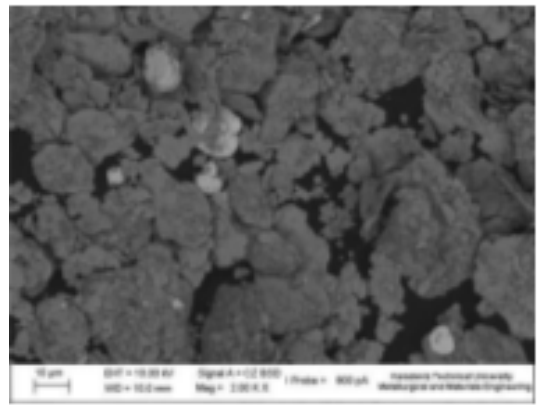

(j)

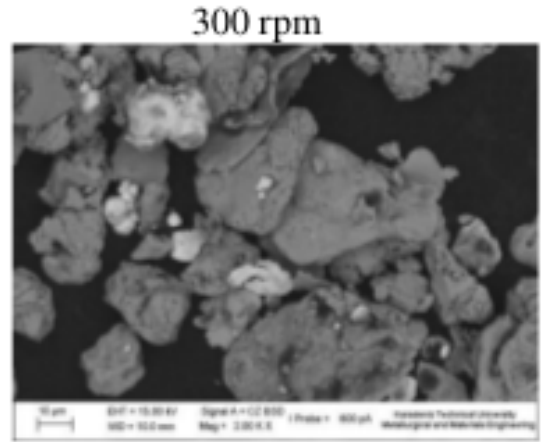

(b)

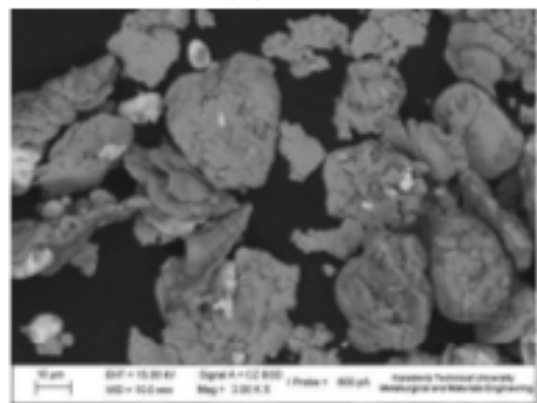

(e)

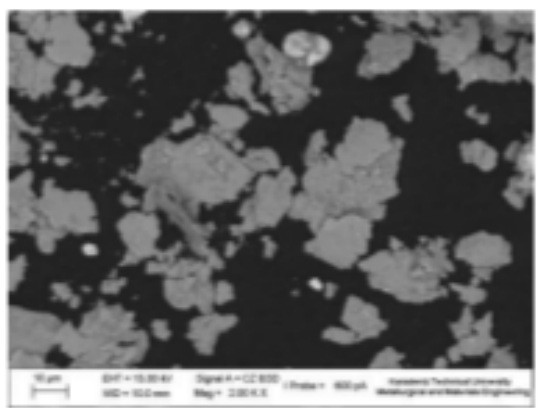

(h)

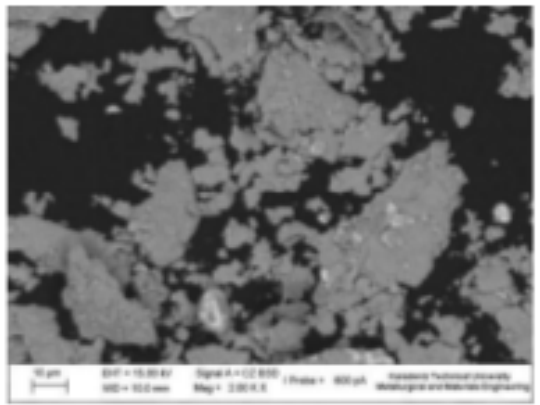

(k)

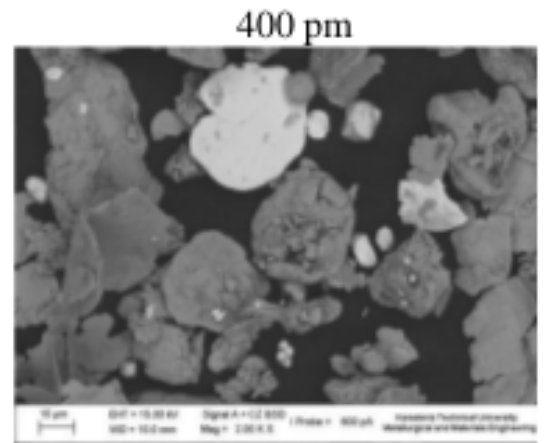

(c)

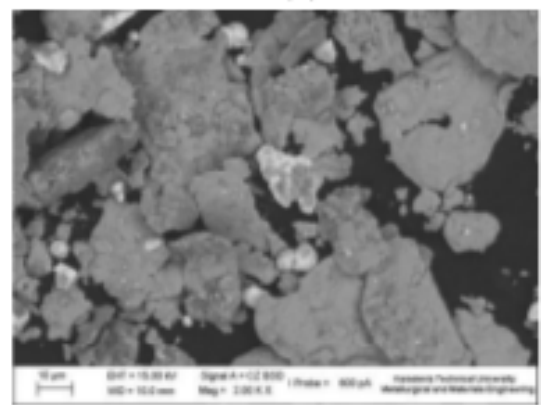

(f)

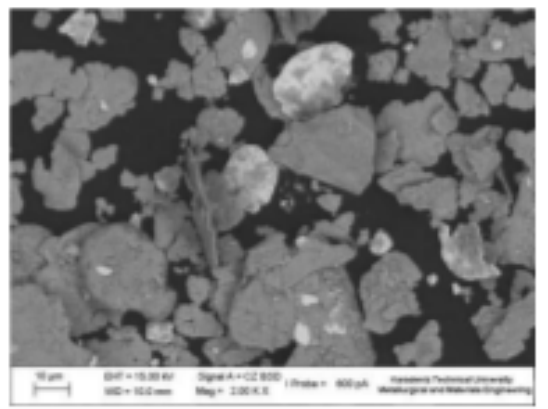

(i)

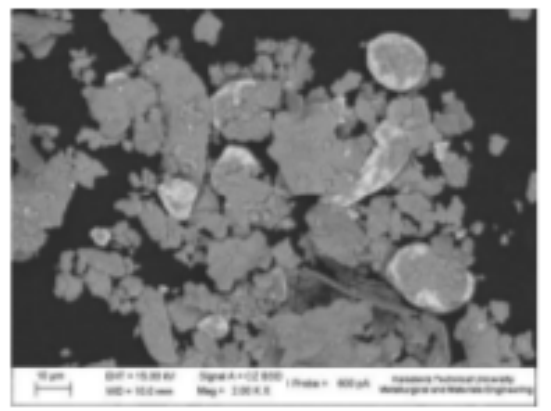

(1)

Fig. 3. Morphologies of the composite powders milled at various speeds for different milling durations (a-b-c) $0.5 \mathrm{~h}$, (d-e-f) $2 \mathrm{~h},(\mathrm{~g}-\mathrm{h}-\mathrm{i}) 4 \mathrm{~h},(\mathrm{j}-\mathrm{k}-\mathrm{l}) 7 \mathrm{~h}$. 
$10 \mathrm{~h}$

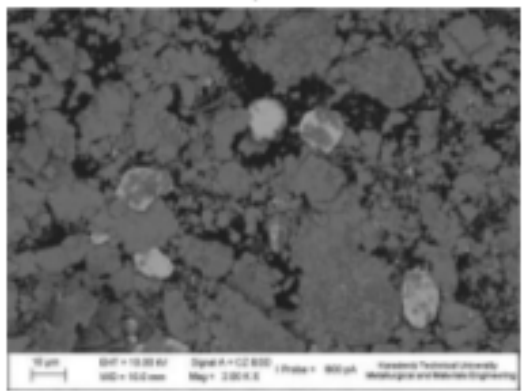

(a)

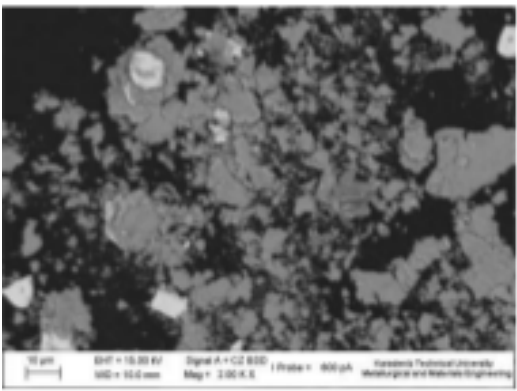

(d)

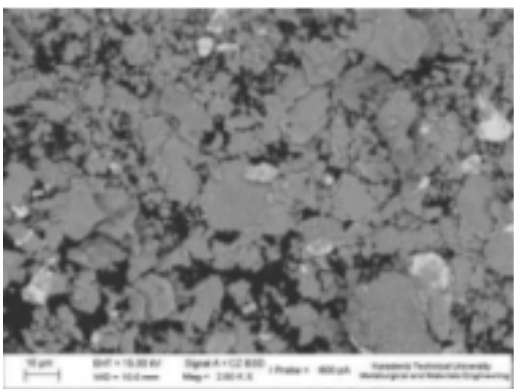

(g)
$16 \mathrm{~h}$

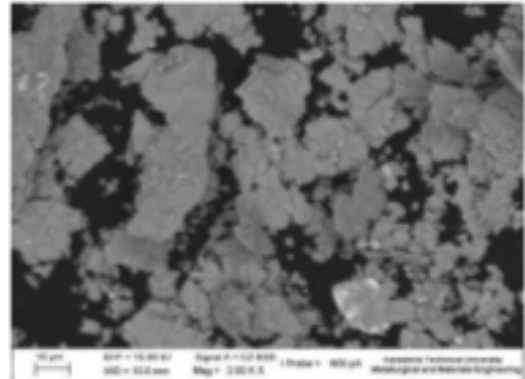

(b)

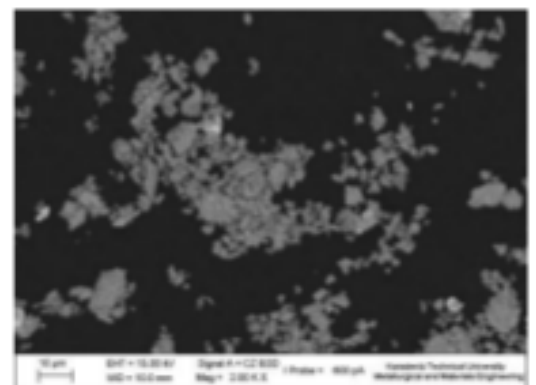

(e)

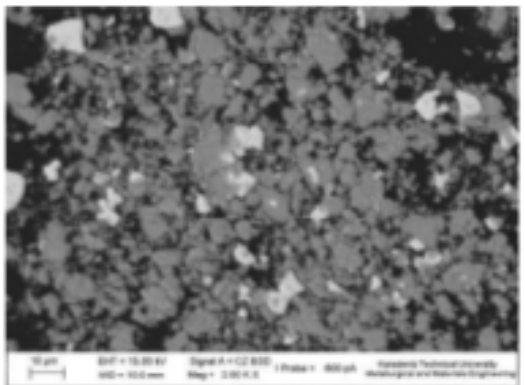

(h)
$25 \mathrm{~h}$

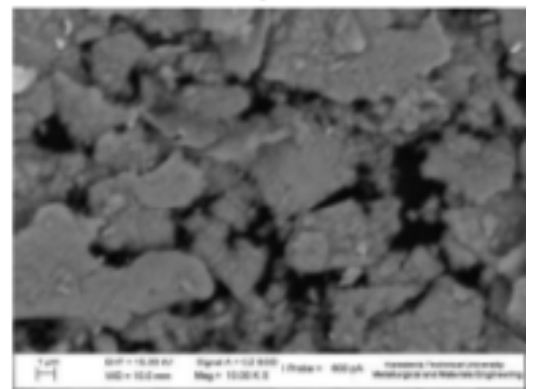

(c)

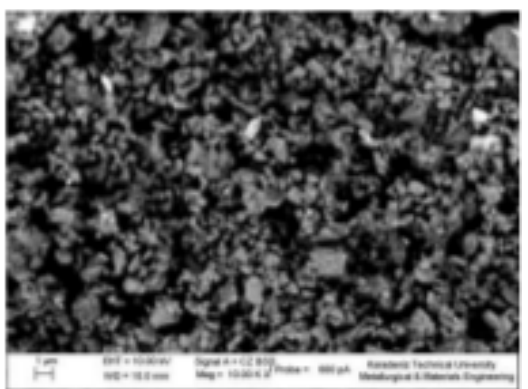

(f)

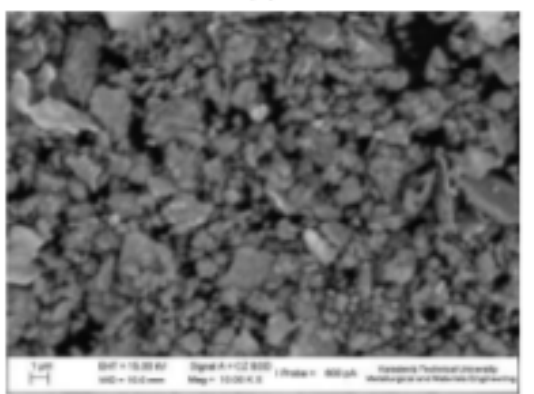

(i)

Fig. 4. Morphologies of the composite powders with respect to milling speed after milled for different milling durations (a-b-c) $200 \mathrm{rpm}$, (d-e-f) $300 \mathrm{rpm},(\mathrm{g}-\mathrm{h}-\mathrm{i}) 400 \mathrm{rpm}$.

In the final stage of milling process, the work hardening of powders was becoming dominant. Brittle W powder particles get fragmented into smaller particles and refined in size. Therefore, the improvement in fracturing efficiency is achieved at the later stages of milling process, which is supported by corresponding SEM image (Fig. 4f). As a consequence, equiaxial morphology was achieved at P2 process, though P3 process was exhibiting semi-equiaxial morphology also (Fig. 4i). Here, care had to be taken to define that very small $\mathrm{W}$ particles behaved in a ductile fashion, during the excessive milling durations, and this caused getting a further reduction in size more difficult or even impossible. Therefore, the limit of comminution of harder $\mathrm{W}$ reinforcement in the softer $\mathrm{Cu}$ matrix is achieved. During the milling of $\mathrm{Cu}$ and $\mathrm{W}$ powders, it has also been observed that some of the $\mathrm{Cu}$ particles get fragmented and embedded into relatively coarse grained $\mathrm{W}$ particles.

The effect of milling speed on the APS of the Cu25W
The shapes of the particles with respect to increasing milling duration, I-Irregular, F-Flake, E-Equiaxial, SE-Semi Equiaxial.

\begin{tabular}{c|c|c|c|c|c|c|c|c}
\hline \hline & Process & \multicolumn{7}{|c}{ Milling time $[\mathrm{h}]$} \\
\cline { 3 - 10 } & & 0.5 & 2 & 4 & 7 & 10 & 16 & 25 \\
\hline Particle & $\mathrm{P} 1$ & $\mathrm{I}$ & $\mathrm{I}+\mathrm{F}$ & $\mathrm{F}$ & $\mathrm{F}$ & $\mathrm{F}+\mathrm{I}$ & $\mathrm{F}+\mathrm{I}$ & $\mathrm{F}+\mathrm{I}$ \\
\cline { 2 - 9 } shape & $\mathrm{P} 2$ & $\mathrm{I}+\mathrm{F}$ & $\mathrm{F}$ & $\mathrm{F}+\mathrm{I}$ & $\mathrm{F}+\mathrm{I}$ & $\mathrm{F}+\mathrm{SE}$ & $\mathrm{SE}$ & $\mathrm{E}$ \\
\cline { 2 - 9 } & $\mathrm{P} 3$ & $\mathrm{I}+\mathrm{F}$ & $\mathrm{F}$ & $\mathrm{F}$ & $\mathrm{F}$ & $\mathrm{F}+\mathrm{I}$ & $\mathrm{F}+\mathrm{SE}$ & $\mathrm{SE}$
\end{tabular}

composite powders with respect to milling time is shown in Fig. 5. The curves in this Figure are plotted using particle size measurements of the milled powders after specified milling durations for each milling speed. The test results containing $d_{50}$ values for all composite powders are presented in Table IV. It can be seen from this table that $4 \mathrm{~h}$ and $7 \mathrm{~h}$ of milling durations are critical points for $\mathrm{P} 2$ and $\mathrm{P} 3$ processes in terms of fracturing. The minimum particle size, namely 0.726 microns is obtained in P2 process after milling for $25 \mathrm{~h}$. On the other hand, there is no significant change on the rate of mi- 
crostructural refinement for $\mathrm{P} 1$ process up to $7 \mathrm{~h}$.

After evaluation of the final particle sizes and morphologies, the optimum milling speed to synthesize Cu25W composite powder was determined as $300 \mathrm{rpm}$ for a given set of experimental conditions. Besides, the optimum speed produced the best result for homogeneous dispersion of $\mathrm{W}$ particles in $\mathrm{Cu}$ matrix.

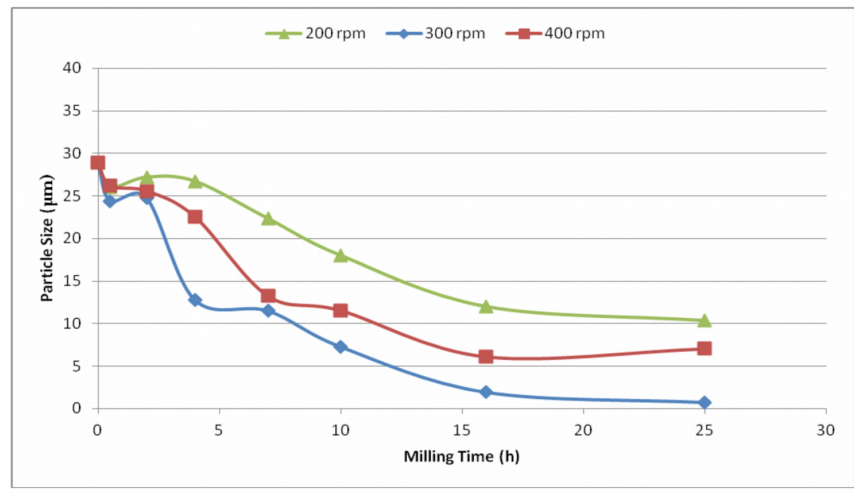

Fig. 5. Particle size vs. milling time graph of $\mathrm{Cu} 25 \mathrm{~W}$ composite powder at different milling speeds.

The APS of the composite powders milled at different speeds.

TABLE IV

\begin{tabular}{c|c|c|c|c|c|c|c|c}
\hline \hline \multirow{4}{*}{$\begin{array}{c}\text { Particle } \\
\text { size }\end{array}$} & Pro- & \multicolumn{7}{|c}{ Milling time $[\mathrm{h}]$} \\
\cline { 2 - 9 }$\left(\mathrm{c}_{50}, \mu \mathrm{m}\right)$ & 0.5 & 2 & 4 & 7 & 10 & 16 & 25 \\
\cline { 2 - 9 }$\left(\mathrm{d}_{50}\right.$ & P1 & 26.045 & 27.206 & 26.723 & 22.365 & 18.042 & 12.034 & 10.395 \\
\cline { 2 - 9 } & P2 & 24.401 & 24.789 & 12.765 & 11.502 & 7.290 & 1.933 & 0.726 \\
\cline { 2 - 9 } & & 25.578 & 22.541 & 13.262 & 11.547 & 6.112 & 7.055
\end{tabular}

\section{Conclusions}

Particle size decreases with increasing milling duration. The minimum particle size ( 0.726 microns) is obtained in the powders milled at $300 \mathrm{rpm}$ speed and $25 \mathrm{~h}$ of milling duration. Rate of welding is dominant with respect to rate of fracturing in the powders milled at $200 \mathrm{rpm}$. Therefore flake morphology remained in the microstructure of the powders milled at $200 \mathrm{rpm}$, even after milled for $25 \mathrm{~h}$. Optimum milling speed is determined to be $300 \mathrm{rpm}$. Equiaxial morphology is achieved by using this milling speed for $25 \mathrm{~h}$. The impact force generated by ball movements is reduced beyond the optimum milling speed. For example, at milling speeds greater than the optimum, W particles are exposed to less deformation and consequently they remain coarser and have flake-like microstructure. Excessive milling caused very small and brittle $\mathrm{W}$ powder particles to behave in a ductile fashion and the powders got to stuck enormously to the inner walls of the grinding chamber. Therefore, the powders are no longer milled beyond $25 \mathrm{~h}$.

\section{Acknowledgments}

This work was supported by the Scientific Research Fund of Karadeniz Technical University (KTUBAP, Project No: 1073).

\section{References}

[1] C. Suryanarayana, Mechanical Alloying and Milling, Marcel Dekker Press, New York 2004.

[2] P.R. Soni, Mechanical Alloying: Fundamentals and Applications, Cambridge International Science, Cambridge 1999 .

[3] C. Suryanarayana, Non-equilibrium Processing of Materials, Pergamon Press, Oxford 1999.

[4] C. Suryanarayana, Prog. Mater. Sci. 46, 1 (2001).

[5] Z. Lin, S. Liu, X. Sun, M. Xie, J. Li, X. Li, Y. Chen, J. Chen, D. Huo, M. Zhang, Q. Zhu, M. Liu, J. Alloy. Compd. 588, 30 (2014).

[6] S. Biyik, F. Arslan, Proc. 6th Int. Powder Metallurgy Conf. and Ex., Ankara 2011, p. 671.

[7] C. Leung, E. Streicher, Proc. 48th IEEE Holm Conf. on Electrical Contacts, Orlando 2002, p. 21.

[8] S. Biyik, M.Sc. Thesis, Karadeniz Technical University, Trabzon 2008.

[9] E. Walczuk, P. Borkowski, S. Ksiezarek, W. Missol, Z. Rdzawski, K. Durst, Proc. 56th IEEE Holm Conf. on Electrical Contacts, Charleston 2010, p. 134.

[10] S. Biyik, M. Aydin, Proc. Int. Porous and Powder Materials Symp. and Ex., Izmir 2013, p. 145.

[11] ASM Handbook: Volume 2: Properties and Selection: Nonferrous Alloys and Special-Purpose Materials, ASM International, Ohio 1990.

[12] H.W. Turner, C. Turner, Copper in Electrical Contacts, CDA Publication, Hertfordshire 1997.

[13] ASM Handbook: Volume 7: Powder Metal Technologies and Applications, ASM International, Ohio 1998.

[14] L. Zhao, Z. Li, Y. Zhou, Proc. 1st Int. Conf. on Electric Power Equipment - Switching Technology, Xi'an 2011, p. 594.

[15] B. Miao, J. Xie, J. He, G. Liu, W. Wang, X. Wang, Proc. 25th Int. Symp. on Discharges and Electrical Insulation in Vacuum, Tomsk 2012, p. 189.

[16] X. Godechot, U. Ernst, L. Dalmazio, S. Kantas, M. Hairour, N. Fadat, Proc. 24th Int. Symp. on Discharges and Electrical Insulation in Vacuum, Braunschweig 2010, p. 206.

[17] O. Guler, E. Evin, J. Mater. Process. Tech. 209 , 1286 (2009).

[18] Y.P. Wang, B.J. Ding, IEEE T. Compon. Pack. T. 22, 467 (1999).

[19] D. Gentsch, K. Gorlt, Proc. 25th Int. Symp. on Discharges and Electrical Insulation in Vacuum, Tomsk 2012, p. 213.

[20] Y. Han, J. Fan, T. Liu, H. Cheng, J. Tian, Int. J. Refract. Met. H. 29, 743 (2011).

[21] M.A. Atwater, D. Roy, K.A. Darling, B.G. Butler, R.O. Scattergood, C.C. Koch, Mat. Sci. Eng. AStruct. 558, 226 (2012).

[22] M. Yusoff, R. Othman, Z. Hussain, Mater. Design. 32, 3293 (2011).

[23] Y. Li, S. Yu, Int. J. Refract. Met. H. 26, 540 (2008).

[24] T. Raghu, R. Sundaresan, P. Ramakrishnan, T.R. Rama Mohan, Mat. Sci. Eng. A-Struct. 304, 438 (2001). 